\title{
Ablation laser femtoseconde pour le dépôt de DLC
}

\author{
A.-S. Loir, F. Garrelie, C. Donnet, F. Goutaland, M. Belin ${ }^{1}$, J.-L. Subtil, \\ R. Le Harzic, F. Rogemond, Y. Ouerdane et P. Laporte
}

\author{
Laboratoire Traitement du Signal et Instrumentation, UMR 5516 du CNRS, \\ Université Jean Monnet, 23 rue du Docteur Paul Michelon, 42023 Saint-Étienne cedex 02, \\ France \\ ${ }^{1}$ Laboratoire de Tribologie et Dynamique des Systèmes, UMR 5513 du CNRS, \\ École Centrale de Lyon, 69134 Ecully cedex, France
}

Résumé. Des films de Diamond-Like Carbon (DLC) ont été déposés, sous vide poussé, par ablation d'une cible de graphite avec un laser Ti : saphir (durée d'impulsion $150 \mathrm{fs}$, fréquence de répétition $1 \mathrm{kHz}$, énergie par impulsion 1,5 mJ, longueur d'onde $800 \mathrm{~nm}$ ) sur substrat standard. Les propriétés de ces couches (structure, adhérence, propriétés nanomécaniques) ont été caractérisées, en fonction des conditions d'élaboration. La caractérisation par spectroscopie Raman de la structure des dépôts obtenus a révélé la présence de diamant nanocristallin au sein d'une matrice amorphe. Le taux d'hybridation $\mathrm{sp}^{3}$ des couches, déterminé à partir des spectres obtenus par XANES ( $X$-ray Absorption Near Edge Spectroscopy), est voisin de $70 \%$. Ces différents résultats sont corrélés avec la caractérisation par imagerie résolue en temps du panache plasma créé par ablation laser femtoseconde.

\section{INTRODUCTION}

Les couches minces de Diamond-Like Carbon (DLC) sont constituées de carbone amorphe, d'hybridation $s p^{2}$ et $s p^{3}$, et présentent des propriétés physiques, chimiques et mécaniques permettant d'utiliser ces revêtements dans de nombreux secteurs d'activités, pour des applications diverses. C'est dans le cadre de la recherche sur la compréhension et l'amélioration des technologies du dépôt de couches que le laser a fait son entrée comme outil alternatif aux techniques de dépôt par voie chimique (Chemical Vapor Deposition, CVD) actuellement les plus utilisées. Le dépôt par ablation laser (Pulsed Laser Deposition, PLD) permet l'obtention de couches de très haute pureté déposées sur des substrats dont la température pendant le dépôt reste proche de l'ambiante, en comparaison des techniques concurrentes. Le domaine biomédical envisage l'utilisation de ces couches comme lubrifiant solide sur prothèses de hanche ou de genou, dans le but d'augmenter la durée de vie de l'implant. Le présent travail consiste à utiliser la technologie PLD en mode femtoseconde pour déposer des couches de carbone susceptibles d'être mises en cuvre dans une application biomédicale. L'intérêt du laser femtoseconde en PLD réside notamment dans la distribution énergétique des espèces du plasma émis lors du bombardement de la cible de graphite par le laser : la présence d'une contribution ionique de haute énergie (de l'ordre du keV) est susceptible de jouer un rôle important dans les mécanismes d'adhésion couche / substrat. Nombreux sont les travaux liés à l'élaboration et à la caractérisation des DLC élaborés par PLD en mode nanoseconde $[1,2]$. Les travaux visant à coupler les paramètres de dépôt de DLC par PLD femtoseconde avec les propriétés des couches sont en revanche très réduits. Dans cet article, nous présentons une étude expérimentale de l'ablation par un laser femtoseconde d'une cible de carbone. Des photographies résolues en temps et spatialement du panache plasma sont présentées, afin d'accéder à la structure du plasma en fonction du domaine spectral étudié. Nous présentons également des caractérisations portant sur la morphologie, la nature des liaisons (hybridations du carbone), la nanostructure, les propriétés nanomécaniques et sur le comportement tribologique de couches minces déposées à différentes fluences laser.

\section{ETUDE DU PANACHE PLASMA - IMAGERIE RESOLUE EN TEMPS}

Un laser saphir : Ti (Concerto, Thalès) de longueur d'onde $800 \mathrm{~nm}$, délivrant une énergie par impulsion de $1.5 \mathrm{~mJ}$ (permettant ainsi d'obtenir des densités de puissance sur la cible de $10^{14} \mathrm{~W} / \mathrm{cm}^{2}$ ) avec une durée d'impulsion de $150 \mathrm{fs}$ a un taux de répétition de $1 \mathrm{kHz}$, est focalisé sur une cible de graphite placée dans une enceinte ultra-vide. Les mesures ont été effectuées sous une pression résiduelle d'air de $1.10^{-5} \mathrm{~Pa}$. Le comportement spatio-temporel du panache plasma créé par ablation laser femtoseconde de la cible de carbone est examiné en utilisant une caméra CCD intensifiée à ouverture rapide calibrée (Hamamatsu). L'imagerie résolue en temps et spatialement a été effectuée avec deux objectifs distincts, l'un transmettant uniquement dans le visible (largeur spectrale : 400-800 $\mathrm{nm}$ ), l'autre transmettant en plus dans l'U.V. (largeur spectrale : $200-800 \mathrm{~nm}$ ) mais avec une transmission moindre dans le visible.

La figure I représente des images du panache plasma à un délai de 400 ns après le début de l'impulsion laser, avec un temps d'intégration du signal de 35 ns. Chaque image correspond à une impulsion laser, la taille de chaque image est 
de $5,6 \times 4,2 \mathrm{~cm}^{2}$. Deux fluences laser ont été examinées : $2,82 \mathrm{~J} / \mathrm{cm}^{2}$ (figure 1(a) et 1 (b)) et $5,18 \mathrm{~J} / \mathrm{cm}^{2}$ (figure 1 (c) et 1(d)), dans deux domaines spectraux différents.
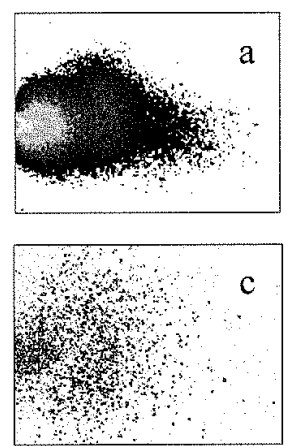
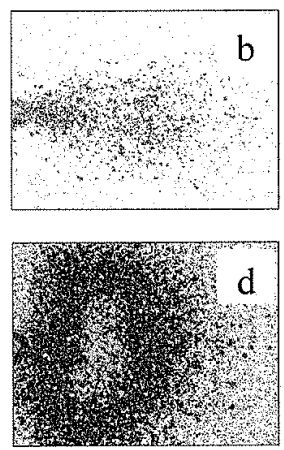

Figure 1 : Images du panache plasma créé par ablation laser femtoseconde d'une cible de carbone, à un délai de $400 \mathrm{~ns}$ après le début de l'impulsion laser (temps d'intégration de chaque image de $35 \mathrm{~ns}$ ), pour une fluence laser de $2,82 \mathrm{~J} / \mathrm{cm}^{2}\left((\mathrm{a})\right.$, (b)), et $5,18 \mathrm{~J} / \mathrm{cm}^{2}((\mathrm{c})$, (d)), à partir de l'émission dans le domaine visible ((a), (c)) et U.V.-Visible ((b), (d))

Plusieurs observations peuvent être effectuées. Tout d'abord, nous pouvons noter une structure différente du panache plasma selon la fluence laser. En effet, les intensités lumineuses détectées sont très différentes, par exemple, dans le domaine spectral visible (figure 1(a) et 1(c)) selon la fluence laser. L'aspect qualitatif du panache peut être corrélé avec l'épaisseur des couches minces déposées dans ces différentes conditions [3].

Par ailleurs, il apparaît ainsi qu'à forte fluence laser $\left(5,18 \mathrm{~J} / \mathrm{cm}^{2}\right)$, l'émission lumineuse du plasma se produit préférentiellement dans 1'U.V. (par comparaison des figures 1(c) et 1 (d)). Compte tenu de la quasi-totale absence de raies spectrales de $\mathrm{C}^{*}$ dans I'U.V. [4], le taux d'ionisation dans le plasma à ce délai après l'impulsion laser est plus élevé à une fluence laser de $5,18 \mathrm{~J} / \mathrm{cm}^{2}$ qu'à une fluence laser de $2,82 \mathrm{~J} / \mathrm{cm}^{2}$. A forte fluence laser, comme nous observons clairement que la composante la plus rapide du plasma émet principalement dans L'U.V., nous pouvons en déduire qu'elle est constituée majoritairement par les ions du plasma.

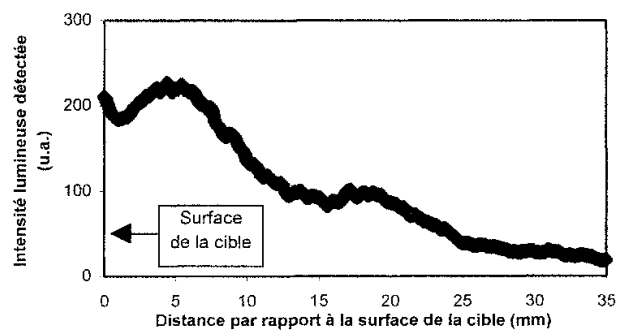

Figure 2 : Evolution de l'intensité lumineuse détectée en fonction de la distance par rapport à la surface de la cible dans le cas de l'objectif visible à une fluence de $2,82 \mathrm{~J} / \mathrm{cm}^{2}$ (correspondant à la figure l(a))

A partir du tracé de l'intensité lumineuse en fonction de la position par rapport à la surface de la cible (figure 2), nous observons clairement deux composantes de vitesse différente comme ont pu les observer précédemment Quian et al. et Banks et al., mais uniquement sur les ions $[5,6]$.

Une troisième composante, constituée de particules de taille micronique ou sub-micronique (escarbilles), apparaît pour des délais de l'ordre de plusieurs dizaines de microsecondes [3].

Des caractérisations AFM (Atomic Force Microscopy) ont été réalisées pour mesurer la rugosité moyenne $\mathrm{R}_{\mathrm{a}}$ et observer l'état de surface des dépôts obtenus sur substrat silicium. Les trois fluences étudiées conduisent à des images comparables. Une rugosité de l'ordre de $1 \mathrm{~nm}$ est déduite de ces images. Cette valeur de $\mathrm{R}_{\mathrm{a}}$ est peu sensible à la présence d'escarbilles, phénomène classique en PLD, dans la mesure où celles-ci sont moins nombreuses et de plus petite taille en mode femtoseconde qu'en mode nanoseconde [3].

\section{CARACTÉRISATIONS STRUCTURALE, NANOMÉCANIQUE ET TRIBOLOGIQUE DES FILMS}

Des couches minces de carbone ont été déposées à différentes fluences laser sur subtrat silicium (100) dopé p. Le détail du dispositif expérimental ainsi que la préparation des échantillons ont déjà été mentionnés par ailleurs [7]. Durant le processus de dépôt, différentes intensités laser peuvent être atteintes en modifiant la focalisation du faisceau sur la cible. 
Les autres paramètres sont maintenus constants de manière à étudier l'effet de la fluence laser sur les propriétés des couches obtenues notamment leurs propriétés tribologiques et nanomécaniques.

Les valeurs des fluences laser utilisées pour les dépôts, ainsi que les propriétés des couches obtenues, sont reportées dans le tableau 1. L'épaisseur des films diminue lorsque la fluence augmente. Ceci s'explique par le fait qu'une augmentation de la fluence, associée à une diminution de la surface d'impact du faisceau sur la cible, induit un équilibre des gradients dans les deux directions de l'expansion du panache de matière ejectée. Ce phénomène conduit à un élargissement de la distribution en épaisseur ( expansion 3D»).

\begin{tabular}{cccccccc}
\hline \hline Echantillon & $\begin{array}{c}\text { Fluence } \\
\left({\left.\mathrm{J} . c m^{-2}\right)}^{-2}\right.\end{array}$ & $\begin{array}{c}\text { Epaisseur } \\
(\mathrm{nm})\end{array}$ & $\begin{array}{c}\text { Vitesse de } \\
\text { dépôt } \\
\left(\mathrm{nm}_{\mathrm{min}}^{-1}\right)\end{array}$ & $\begin{array}{c}\text { Pourcentage } \\
\text { de carbone } \\
s p^{3}(\%)\end{array}$ & $\begin{array}{c}\text { Dureté } \\
(\mathrm{GPa})\end{array}$ & $\begin{array}{c}\text { Module } \\
\text { d'Young } \\
(\mathrm{GPa})\end{array}$ & $\begin{array}{c}\text { Contraintes } \\
\text { résiduelles } \\
(\mathrm{GPa})\end{array}$ \\
\hline $\mathbf{A}$ & 1,35 & $250-300$ & 50 & 71 & $20 \pm 2,3$ & $240 \pm 29$ & $-0,81$ \\
$\mathbf{B}$ & 2,82 & $120-150$ & 30 & 73 & $25 \pm 2,5$ & $260 \pm 10$ & $-1,85$ \\
$\mathbf{C}$ & 5,18 & $85-95$ & 20 & 70 & $18 \pm 1,3$ & $210 \pm 13$ & $-2,83$ \\
\hline
\end{tabular}

Tableau 1 : Caractéristiques et propriétés des films obtenus

La nature du DLC, en terme de proportions d'hybridations $s p^{2}$ et $s p^{3}$, est un facteur important reliant le procédé de dépôt aux propriétés. La caractérisation du rapport $s p^{2} / s p^{3}$ est cependant délicate pour les carbones amorphes. Les couches ont été caractérisées conjointement par spectroscopie Raman et par spectroscopie XANES. La figure 3 (a) présente le spectre Raman de la couche obtenue à une fluence laser de $2,82 \mathrm{~J}_{\mathrm{cm}}{ }^{-2}$. Les deux autres fluences étudiées conduisent à des spectres Raman semblables.

On observe les deux bandes larges, traditionnellement notées $D$ et $\mathrm{G}$, respectivement centrées à 1340 et $1500 \mathrm{~cm}^{-1}$ et caractéristiques d'une structure graphitique désordonnée [8]. On notera cependant la présence systématique d'un pic fin centré à $1140 \mathrm{~cm}^{-1}$, dont l'interprétation est très discutée dans la littérature. Plusieurs auteurs attribuent ce pic à des nanocristaux de diamant $[9,10]$ ou à des cristallites de diamant dispersés dans le réseau de carbone amorphe [11]. Cette interprétation a été repoussée par Ferrari et al. [12] qui attribuent ce pic à un effet de résonance dû à des segments de polymère acétylènique. Dans notre cas, cette dernière interprétation ne paraît pas acceptable. D'une part, en effet, les DLC obtenus sont exempts d'hydrogène. D'autre part ce pic est observé aux deux longueurs d'onde d'analyse RAMAN sélectionnées (488 et $514 \mathrm{~nm}$ ), avec une finesse supérieure à celle caractéristique d'un pic de résonance.

La quantification de taux d'hybridation $s p^{3}$ n'est pas précise en spectrométrie Raman dans le visible, dans la mesure où la section efficace de la contribution $s p^{3}$ est environ 50 fois plus faible que la section efficace de la contribution $s p^{2}$. La spectroscopie XANES réalisée sur les dépôts obtenus à différentes fluences permet de remonter au taux d'hybridation $s p^{3}$ des atomes de carbone par intégration des pics $\pi^{*} s p^{2}$ et $\sigma^{*} s p^{3}$ du graphite et du diamant respectivement $[13,14]$. A partir des spectres (figure 3 (b)), on déduit un pourcentage d'atomes de carbone hybridés $s p^{3}$ de l'ordre de $70 \%$. La fluence, dans la gamme étudiée, ne semble pas affecter de manière significative la nature du DLC en terme d'hybridation du carbone. De plus, ce résultat est cohérent avec la présence de nanocristaux de diamant révélés par spectrométrie Raman. La comparaison des spectres des DLC avec ceux du graphite et du diamant met en évidence que les DLC déposés par ablation laser femtoseconde se caractérisent par une structure moléculaire particulièrement complexe, révélée par la présence de bandes intermédiaires à $286,4,287,3$ et $288,4 \mathrm{eV}$. L'interprétation exacte de l'origine de ces bandes n'est actuellement pas totalement élucidée.

(a)

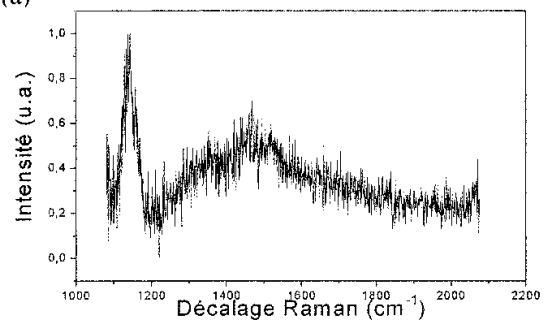

(b)

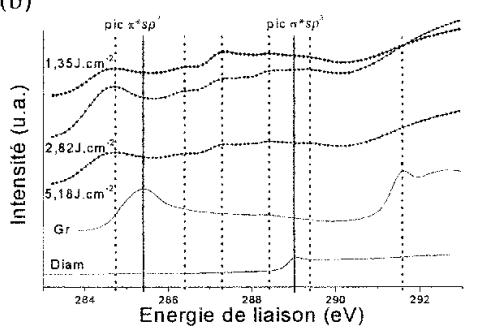

Figure 3 : (a) Spectre Raman de la couche déposée à une fluence laser de $2,82 \mathrm{~J} . \mathrm{cm}^{-2}$

(b) Spectres XANES du seuil $\mathrm{K}$ du Carbone $1 \mathrm{~s}$ des films déposés à une fluence de $1,35 \mathrm{~J} . \mathrm{cm}^{-2}, 2,82 \mathrm{~J} . \mathrm{cm}^{-2}$ et $5,18{\mathrm{~J} . \mathrm{cm}^{-2}}^{-2}$ spectres de référence du graphite (Gr) et du diamant (Diam) déposé par Chemical Vapor Deposition (CVD)

Les contraintes résiduelles sont, de manière très significative, faibles par rapport aux valeurs observées dans des couches DLC non dopées et sans sous-couches d'accrochage. Dans la gamme de fluences laser étudiée, on observe en 
effet (tableau 1) des valeurs au plus égales à $2,83 \mathrm{GPa}$ (en compressif), ce qui est inférieur aux niveaux de contraintes observées par exemple sur des couches ta- $C$ obtenues par ablation laser en mode nanoseconde [1]. Ce résultat, qui peut s'expliquer par la distribution énergétique du plasma issu de l'interaction laser femtoseconde-matière, est en faveur d'une meilleure adhérence de la couche, en particulier sous sollicitation tribologique [8]. Ces faibles niveaux de contraintes résiduelles, si elles sont confirmées par des expériences complémentaires, représenteraient un avantage majeur de l'ablation femtoseconde, par rapport au mode nanoseconde. Perrière et al. ont également observé une tendance similaire, en comparant des couches de ZnO déposées par ablation laser femtoseconde et nanoseconde [15]. Les duretés et les modules d'élasticité en fonction de la profondeur d'indentation ont été mesurés pour les trois fluences laser déjà mentionnées. Les résultats sont reportés dans le tableau 1. Les valeurs atteignent un maximum pour une fluence de 2,82 $\mathrm{J} \mathrm{cm}^{-2}$ avec une dureté de $25 \mathrm{GPa}$ et un module d'Young de $260 \mathrm{GPa}$. Ces résultats sont relativement modestes comparés à ceux obtenus par ablation laser en régime nanoseconde [16,17]. Cependant, ces valeurs sont tout à fait conformes à celles de la littérature [18] compte tenu du taux d'hybridation $s p^{3}$ mesuré dans nos couches.

Les couches obtenues se caractérisent par des coefficients de frottement fäbles $(0,1-0,2)$ et stables. Les premiers essais d'usure en longue durée ( 50000 cycles), réalisés pour la fluence de $2,82 \mathrm{~J}_{\mathrm{cm}} \mathrm{cm}^{-2}$, ont conduit à un coefficient d'usure de $1,6 \cdot 10^{-8} \mathrm{~mm}^{3} \cdot \mathrm{N}^{-1} \cdot \mathrm{m}^{-1}$. Cette valeur classe les couches carbone réalisées par ablation laser femtoseconde parmi les dépôts de DLC à haute résistance à l'usure publiées dans la littérature [19].

\section{CONCLUSION}

Ce travail vise à coupler les paramètres de dépôt de DLC élaborés par PLD femtoseconde avec les propriétés des couches réalisées. Le diagnostic du panache plasma met clairement en évidence la présence de deux composantes de vitesse différente. Cette étude montre également que les premières couches obtenues sont constituées majoritairement d'atomes de carbone hybridés $s p^{3}$ et la présence de nanocristaux de diamant au sein de ces couches. Les différentes caractérisations permettent de classer ces couches minces parmi les matériaux à haute résistance à l'usure. Les perspectives de ces travaux s'orienteront principalement vers l'étude plus approfondie des caractéristiques du panache plasma en fonction de la fiuence laser puis vers les dépôts sur substrats biocompatibles de plus grande dimension et sur des pièces réelles.

\section{Remerciements}

La présente étude est réalisée avec le soutien financier de la Région Rhône-Alpes (Thématiques Prioritaires), du Conseil Général de la Loire et avec le concours de l'Union Européenne (Fonds FEDER) dans le cadre du Programme de Reconversion Régionale et Sociale 1997-1999. Les auteurs remercient également S. Reynaud (LTSI, Saint-Etienne) pour les analyses AFM, S. Pavan et J.-L. Loubet (LTDS, Lyon) pour les caractérisations par nanoindentation et M. Kasrai (CSRF, Wisconsin-Madison) pour l'étude XANES.

\section{Références}

[1] Voevodin A.A., Donley M.S. et Zabinski I.S., Surf. Coat. Technol. 92 (1997) 42-49.

[2] Chrisey D.B. et Hlubler C.K., Pulsed Laser Deposition of Thin Film (Editions Wiley \& Sons, New York, 1994).

[3] Garrelie F., Loir A-S., Goutaland F., Donnet C., Le Harzic R., Angleraud B., Ouerdane Y, el Laporte P., « Diamond-Like Carbon deposited by femtosecond pulsed laser ablation: evidence of nanocrystalline diamond », Conférence High Power Laser $\Lambda$ blation IV, Taos (NM, USA), 21-26 Avril 2002, à paraittre dans SPIE Proceedings.

[4] Striganov A.R. et Sventitskii N.S., Tables of Spectral Lines of Neutral and lonized Atoms (IFI/Plenum, New York, Washington, 1968).

[5] Qian F., Craciun V., Singh R.K., Dutta S.D. et Pronko P.P., J. Appl. Phys. 86 (1999), $2281-2290$.

[6] Banks P.S., Dinh L., Stuart B.C., Feit M.D., Komashko A.M., Rubenchik A.M., Perry M.D. et McLean W.. Appl. Phys. A 69 (1999), S347-S353.

[7] Garrelie F., Loir A-S., Donnet C., Rogemond F., Le I Iarzic R., Belin M., Audouard E. et Laporte P., Surf. Coat. Technol. (2002) In Press.

[8] Wei Q., Sankar J., Narayan J., Surf. Coat. Technol. 146/147 (2001) 250-257.

[9] Schwan J., Ulrich S., Roth H., Ehrhardt H., Silva S.R.P., Robertson J., Samlenski R., Brenn R., J. Appl. Phys. 79 n 3 (1996) 1416-1422.

[10] Sharda T., Soga T., Jimbo T., Umeno M., Diamond Relat. Mater. 10 (2001) 1592-1596.

[11] Silva S.R.P., Amaratunga G.A.J., Salje E.K.H., Knowles K.M., Journal of Material Science 29 (1994) 4962-4966.

[12] Ferrari A.C., Robertson J., Phys Rey B 63 (2001) 121405(R).

[13] Pappas D.L., Saenger K.L., Bruley J., Krakow W. et Cuomo J.J, J. Appl. Phys. 71 n$^{\circ} 11$ (1992) 5675-5684

[14] Koivusaari K.I., Levoska I. et Leppävuori S., J. Appl. Phys. 85 nº5 (1999) 2915-2920.

[15] Perrière J., Millon E., Seiler W., Boulmer-Leborgne C., Craciun V., Albert O., Loulcrgue J.C. et Etchepare J., J. Appl. Phys 91 $n^{\circ} 2(2002) 690-696$.

[16] Bonelli M., Fioravanti A.P., Miotello A. et Ossi P.M., Europhys. Lett. 50 n $^{\circ} 4$ (2000) 501-506.

[17] Voevodin A.A. et Donley M.S., Surf. Coat. Technol. 82 (1996) 199-213.

[18] Robertson J., Mater. Sci and Eng. R: Reports, 37 (2002) 262.

[19] Erdemir A. et Donnet C., Modern Tribology Handbook (B. Bhushan CRC Press LLC, Boca Raton, 2000$) 2$ chpt 24 p. 871 